Mens

revue d'histoire intellectuelle de l'Amérique française

\title{
Sur l'historiographie de la télévision au Québec et le pesant récit de la Révolution tranquille
}

\section{Frédéric Demers}

Volume 3, numéro 2, printemps 2003

Regards sur la Révolution tranquille

URI : https://id.erudit.org/iderudit/1024644ar

DOI : https://doi.org/10.7202/1024644ar

Aller au sommaire du numéro

Éditeur(s)

Centre de recherche en civilisation canadienne-française

ISSN

1492-8647 (imprimé)

1927-9299 (numérique)

Découvrir la revue

Citer cette note

Demers, F. (2003). Sur l'historiographie de la télévision au Québec et le pesant récit de la Révolution tranquille. Mens, 3(2), 233-267.

https://doi.org/10.7202/1024644ar

\section{Résumé de l'article}

Les historiens québécois entretiennent une attitude contradictoire face à l'arrivée de la télévision dans la province en 1952. Présentant cet événement comme un tournant dans le passage du Québec à la modernité, ils n’y consacrent pourtant aucun effort de recherche soutenu et se contentent de répéter, d'une synthèse à l'autre, les mêmes généralités à son sujet. De manière isolée, quelques chercheurs ont toutefois proposé des pistes de réflexion et fourni certaines clefs pour mieux comprendre et évaluer la signification historique de ce phénomène. Il serait pertinent que la communauté historienne y fasse écho, quoique cela impliquerait peut-être de réévaluer de fond en comble le récit hégémonique de la Révolution tranquille et de la naissance du Québec moderne. 


\title{
SUR L'HISTORIOGRAPHIE DE LA TÉLÉVISION AU QUÉBEC ET LE PESANT RÉCIT DE LA RÉVOLUTION TRANQUILLE ${ }^{1}$
}

\author{
Frédéric Demers \\ CELAT \\ Université Laval
}

\section{RÉSUMÉ}

Les historiens québécois entretiennent une attitude contradictoire face à l'arrivée de la télévision dans la province en 1952. Présentant cet événement comme un tournant dans le passage du Québec à la modernité, ils n'y consacrent pourtant aucun effort de recherche soutenu et se contentent de répéter, d'une synthèse à l'autre, les mêmes généralités à son sujet. De manière isolée, quelques chercheurs ont toutefois proposé des pistes de réflexion et fourni certaines clefs pour mieux comprendre et évaluer la signification historique de ce phénomène. Il serait pertinent que la communauté historienne y fasse écho, quoique cela impliquerait peut-être de réévaluer de fond en comble le récit hégémonique de la Révolution tranquille et de la naissance du Québec moderne.

\section{ABSTRACT}

Quebec's historians hold a contradictory attitude towards the 1952 arrival of television in the Province. While they present this event as a turning point in Quebec's modernisation, they devote little or no sustained research to television's early impact on French Canadian society. Consequently, the same bland 
pleasantries on television's arrival can be found in all the general surveys of Quebec history. Overall, only a select few French Canadian scholars have offered research avenues and keys to better understand television's historical significance. However, these avenues must be pursued, even if they challenge the traditional narrative of the Quiet Revolution and the birth of modern Quebec.

En 1962, l'année où la télévision fêtait ses dix années d'existence au Canada, le dramaturge Marcel Dubé fut invité à présenter un bilan personnel de cette décennie de télédiffusion dans une perspective québécoise et canadienne-française ${ }^{2}$. Dans son allocution, Dubé suggéra que la télévision avait dressé « un inventaire de nos forces, de nos richesses, de nos talents ; un inventaire de notre âme aussi, un inventaire de nos sentiments $^{3} »$. Pour lui, la télévision avait activement participé à la définition de l'identité collective. Il insista également sur son rôle d'instrument d'acquisition d'une maturité politique et culturelle après la longue indigence intellectuelle imposée par le carcan clérico-nationaliste, après aussi le mépris professé envers ceux qui avaient des idées différentes. La télévision, estimait-il, avait directement développé le goût des débats et elle était rapidement devenue un enjeu, un instrument à maittriser pour les contestataires de l'ordre social.

Il n'est pas surprenant que Dubé ait proposé un tel bilan. Ayant activement travaillé pour la télévision dans les premières années et manquant de recul pour bien évaluer le phénomène télévisuel, le dramaturge magnifia en quelque sorte l'impact d'un fait de société (au sens large) qu'il avait lui-même contribué à créer.

Vingt ans après Marcel Dubé, et sans mettre de gants blancs, le professeur Gérard Laurence invitait les chercheurs à s'engager dans le champ des études sur l'histoire de la télévision. " On a toujours attribué un certain rôle au nouveau média dans le déclenchement de la Révolution tranquille, mais sans 
pousser plus loin l'analyse, constatait-il. Il n'est plus possible, aujourd'hui, de faire abstraction du phénomène de la télévision dans l'histoire récente du Québec ou de se contenter d'affirmations élémentaires ${ }^{4}$.»

Tant le bilan tracé par Marcel Dubé que les lacunes constatées par Gérard Laurence constituaient un appel à l'historien afin qu'il se penche sur le rôle de la télévision dans l'évolution du Québec des années cinquante. On sait à quel point la réflexion sur l'identité collective préoccupe les intellectuels d'ici. On sait également que le grand récit national contemporain des Québécois d'héritage canadien-français a fait de la Grande Noirceur un de ses principaux référents identitaires. Dans la mesure où la question des répercussions de la télévision naissante sur l'évolution de la société québécoise rejoint l'un et l'autre de ces éléments, on aurait pu s'attendre à ce que l'historien investisse le champ des études sur la télévision. Et pourtant, quarante ans après l'appel lancé par Dubé, vingt ans après celui répété par Laurence, les connaissances historiques acquises en rapport avec l'existence et les effets de la télévision à cette époque n'excèdent pas de beaucoup le niveau des généralisations et des approximations.

Sur la place de la télévision dans la production savante au Québec, on remarque principalement deux choses. Tout d'abord, les études sur un aspect ou un autre de la télévision se sont multipliées depuis un quart de siècle, mais pas dans une perspective historienne (ou historique) : ce sont les spécialistes des communications, et plus récemment les littéraires, qui s'y intéressent, et c'est pour étudier les contenus des émissions d'aujourd'hui, les mécanismes de programmation, les règles propres à l'industrie télévisuelle ou encore la réception des émissions. Au Québec, il n'existe à l'heure actuelle aucune recherche soutenue qui soit consacrée à l'histoire de la télévision dans ses dimensions sociale et politique. À ce jour, la meilleure 
source d'information (produite dans une perspective canadienne) reste un ouvrage publié en 1990 par un historien de l'Université de Toronto, Paul Rutherford ${ }^{5}$. Les grandes synthèses historiennes et les nombreux ouvrages qui abordent les années cinquante sous un aspect ou un autre n'accordent qu'une place minimale à la télévision. De plus, lorsqu'ils en parlent, c'est de manière assez furtive, superficielle, et en recourant généralement aux lieux communs d'usage. La remarque que faisait Laurence il y a deux décennies vaut donc encore. La deuxième chose qu'on note, en opposition à la première, c'est que lorsque les ouvrages d'histoire font référence à la télévision, ils reprennent souvent à leur compte les grandes lignes du bilan de Marcel Dubé. Il y a donc là une contradiction manifeste : l'historien admet l'importance de la télévision et lui reconnaît un rôle clé dans le processus de modernisation du Québec, mais il en parle d'une manière presque anecdotique, s'abstenant - sauf exception - de creuser un tant soit peu cette question.

C'est ce problème que nous souhaitons aborder dans les pages qui suivent. Ce faisant, notre ambition première est d'attirer l'attention sur une situation, non d'attaquer ou de dénoncer. L'historiographie dont nous rendons compte concerne deux catégories d'ouvrages, soit celle des synthèses d'histoire nationale du Québec parues depuis un quart de siècle environ et celle des livres plus ciblés, abordant par exemple, dans une perspective large, le nationalisme, les idéologies ou encore les années cinquante ${ }^{6}$.

\section{Sur l'état et les limites de l'historiographie de la télévision au Québec}

Disons-le d'emblée : jusqu'à ce jour, parmi tous les auteurs de synthèses d'histoire du Québec, c'est une anglophone, Susan Mann Trofimenkoff, qui manifeste le plus d'in- 
térêt pour la signification de l'avènement de la télévision dans la province ${ }^{7}$. Désireuse de ne pas s'en tenir aux idées préconçues, elle est la seule qui se préoccupe réellement de l'intégrer au tableau qu'elle peint de l'évolution sociale et politique du Québec d'alors. En comparaison avec elle, les autres auteurs de synthèses d'histoire du Québec destinées à des clientèles universitaires et collégiales, voire au grand public, se montrent fort discrets sur cette question.

Nous pouvons regrouper leur production en trois ensembles. Le premier inclut les auteurs qui ne disent rien de la télévision et, ce faisant, lui nient toute influence sociopolitique et historique. Réunissant les auteurs qui concèdent à la télévision une certaine importance politique et idéologique (pour ne pas dire une importance certaine), le second ensemble englobe le plus grand nombre de titres. Le dernier, enfin, comprend les historiens qui prêtent à la télévision naissante quelque valeur symbolique ou influence, mais sans nécessairement en faire un agent efficace de contestation politique et idéologique. Il serait donc à situer entre les deux ensembles précédents.

$\mathrm{Au}$ sein du premier ensemble, la cause est entendue d'avance : l'arrivée de la télévision au Québec est historiquement insignifiante. Interrogé à titre de spécialiste des années cinquante par Gilles Gougeon de la télévision de Radio-Canada - quelle ironie, tout de même! - pour son Histoire $d u$ nationalisme québécois, Richard Desrosiers ne mentionne pas l'avènement de la télévision et n'émet donc aucune hypothèse sur ce que cela aurait pu signifier pour la diffusion du néo-nationalisme (à la manière d'André Laurendeau) et de l'antinationalisme (à la manière de Cité libre) ${ }^{8}$. Le mutisme de Desrosiers prolonge en quelque sorte celui du vaste triptyque collectif sur les idéologies au Canada français entre 1940 et $1976^{\circ}$. Y aurait-il, après 1952, quelque lien à faire entre l'existence de la télévision et la diffusion de certaines idéologies au sein de la province de Qué- 
bec ? La question ne s'est apparemment pas posée. Une même absence d'intérêt — ou de vision — pour le médium télévisuel comme objet d'histoire caractérise les contributions à un important colloque qui s'est tenu à Montréal, ces dernières années, sur le thème de la société duplessiste ${ }^{10}$. Le rôle historique qu'a pu jouer la télévision n'a aucunement interpellé les participants à cette rencontre dont l'objectif annoncé était pourtant de saisir «toute la complexité de la société québécoise de cette période ${ }^{11} »$.

Quoi qu'il en soit, l'absence d'intérêt pour la télévision n'est jamais aussi déroutante que chez Jacques Lacoursière. On sait à quelle fréquence et avec quel succès l'historien-vulgarisateur a lui-même beaucoup utilisé la télévision pour diffuser et encourager la connaissance du passé du Québec. Il est donc surprenant de constater que Lacoursière ne souffle pas mot de la naissance de la télévision dans le corps du texte de deux des trois synthèses qu'il a signées, à savoir l'Histoire populaire du Québec et Canada-Québec, 1534-2000 (celle-ci avec Jean Provencher et Denis Vaugeois) ${ }^{12}$. Simplement, à la fin du chapitre de l'Histoire populaire sur les années cinquante, Lacoursière nous propose une vignette montrant une famille qui regarde la télévision, tandis que dans Canada-Québec, la télévision n'existe qu'à travers une mention furtive dans la chronologie placée en annexe.

Plus vaste que les deux autres, le second ensemble est celui où s'exprime le discours historien dominant sur la télévision. Pour l'essentiel, les auteurs qui le composent creusent les sillons autrefois tracés par Marcel Dubé en recourant aux mêmes images et métaphores, et dans des mots parfois identiques.

Largement reconnue comme la meilleure des synthèses québécoises, l'Histoire du Québec contemporain de Paul-André Linteau, René Durocher, Jean-Claude Robert et François Ricard 
compte un chapitre intitulé "L'avènement du petit écran ». Malgré ce titre, les auteurs ne consacrent qu'environ deux pages de texte à la naissance de la télévision à proprement parler et condensent en un seul paragraphe son impact sur le renouveau des mentalités et des modes usuels de perception et de projection de soi au sein de la population canadienne-française. Ils estiment néanmoins que cette invention « joue un rôle crucial dans l'évolution de la société québécoise " au cours des années cinquante ${ }^{13}$. Elle y parvient en diffusant des idées nouvelles et la critique sociale, en favorisant l'uniformisation à l'échelle de la province des valeurs et des mentalités, et en contribuant à la prise de conscience par les Canadiens français du Québec qu'ils forment "une société distincte, possédant des traits et des besoins collectifs qui lui sont propres ${ }^{14} »$. Cela dit, les auteurs restent prudents dans leurs évaluations. «S'il est un domaine où l'avènement de la télévision a l'effet d'une véritable révolution, écrivent-ils comme si un doute subsistait dans leur esprit, c'est celui du divertissement et de la culture de consommation ${ }^{15}$. » Et de fait, la plus grande partie de ce chapitre, malgré son titre, concerne le cinéma, le théâtre, la radio, la presse à grand tirage, le roman populaire et les cabarets plutôt que la télévision à proprement parler.

La prudence de Linteau et compagnie fait défaut ailleurs, où l'apport idéologique de la télévision, tenu pour évident, n'est nullement débattu. Dans le texte de Fondements historiques du Québec contemporain de Gilles Laporte et Luc Lefebvre, la télévision passe en coup de vent, mais non sans que la thèse de la fenêtre et du miroir soit dûment reprise sans nuance ${ }^{16}$. L'Histoire du Québec d'bier à l'an 2000, d'Yves Tessier, arpente les mêmes sentiers battus: "condition importante à l'origine de la révolution tranquille ", fenêtre, miroir, bris de l'isolement, etc., telles sont les caractéristiques de la jeune télévision au Qué$\mathrm{bec}^{17}$. Dans son Bilan du nationalisme au Québec, Louis Balthazar 
érige la télévision naissante au rang de « canal d'expression privilégié pour la nouvelle intelligentsia canadienne-française » qui conteste l'ordre social et le nationalisme traditionnel défendus par Duplessis et l'Église catholique. Or, en dépit de cette opinion assurée, il limite son propos à un paragraphe de généralités ${ }^{18}$. Gérard Bouchard, dans Genèse des nations et cultures $d u$ Nouveau Monde, fait de la télévision l'une de ces « données circonstancielles » qui ont préparé la Révolution tranquille, mais sans pousser sa réflexion plus en avant ${ }^{19}$. À peine plus loquace que ne l'est Bouchard, ou qu'il ne l'était lui-même dans ses précédents ouvrages où il n'en disait rien, Jacques Lacoursière, dans Une histoire du Québec, évacue la question en une trentaine de mots : la télévision constitue « un élément de changement dans l'opinion publique » en faisant pénétrer «le monde » chez les gens $^{20}$. Pour sa part, Denis Monière, dans Le développement des idéologies au Québec, expédie en une phrase le rôle de la télévision dans le processus de changement de mentalité du Québec français de l'après-guerre, et pourtant, comme d'autres, il lui accorde un grand crédit. Après 1952, soutient-il, « l'ère de la télévision amplifiera cette prise de conscience [apparue à la suite de la guerre] en élargissant les cadres culturels et en oxygénant les schèmes de réflexion ${ }^{21} »$. Les conférenciers invités au grand colloque sur la Révolution tranquille qui s'est tenu à l'UQAM au printemps 2000 ne se sont pas montrés plus curieux ${ }^{22}$. Quelques-unes des communications ont illustré le paradoxe habituel, soulignant l'importance de la télévision en tant que relais des idées qui préparèrent la Révolution tranquille, mais n'en appelant pas pour autant au dépassement des lieux communs, clichés et formules prémâchées d'usage ${ }^{23}$.

D'autres auteurs, tout en s'inscrivant dans le même registre sémiotique, abordent l'avènement de la télévision avec encore plus de détachement (si l'on peut dire). C'est entre autres le cas de Jean Hamelin et Jean Provencher dans leur Brève his- 
toire du Québec. Comme dans un haussement d'épaules chargé d'indifférence, ils concluent un paragraphe sur les mouvements de contestation et les forces de changement dans le Québec des années cinquante par la phrase suivante: "Sans compter que l'avènement de la télévision en 1951 (sic) commence à bouleverser les croyances et les comportements traditionnels ${ }^{24}$." Cette banalisation trouve son aboutissement dans la Brève histoire socio-économique du Québec de John A. Dickinson et Brian Young. Chez eux, la télévision n'est même pas différenciée de la radio. La Société Radio-Canada, se contentent-ils d'écrire dans les trois dernières phrases du chapitre sur les années 19301960, tend, par les deux moyens dont elle dispose, «à inculquer aux francophones un système de valeurs urbaines » et essaie d'ouvrir « les horizons des Québécois » avec des émissions d'affaires publiques comme Point de mire, laquelle offre une perspective québécoise à l'actualité internationale ${ }^{25}$. La Société réussit-elle ? Poursuit-elle d'autres objectifs ? Ses programmes ont-ils des effets additionnels ? Par rapport à la radio, la télévision a-t-elle des particularités distinctes? Jusqu'à quel point peut-on établir un lien entre elle et le climat de contestation qui caractérise le Québec de l'après-guerre? Que comprennent et que retiennent ceux qui la regardent? Ces questions n'effleurent pas l'esprit de Dickinson et Young, pas plus qu'elles ne semblent intéresser Balthazar, Bouchard, Hamelin, Lacoursière, Linteau, Provencher et tous les autres.

Et si l'avènement de la télévision au Québec, sans être insignifiant, n'avait pas le sens que lui donne, même en peu de mots, le discours dominant ? C'est cette hypothèse commune qui réunit les historiens du troisième ensemble.

Responsable de la période 1939-1960 pour l'Histoire du Québec dirigée par Jean Hamelin, Richard Jones n'évoque la naissance, l'existence et l'impact de la télévision que d'une manière anecdotique dans quelques petites phrases enfouies en fin de 
chapitre. L'auteur décrit la télévision comme un instrument d'américanisation potentielle, puis montre, en citant tour à tour Point de Mire et le téléroman La famille Plouffe, que la pratique du journalisme atteint une nouvelle maturité et que les préoccupations artistiques glissent de la vie rurale vers le monde ouvrier $^{26}$. Prenant de semblables distances par rapport au discours dominant, l'Histoire du Québec d'Yves Bourdon et Jean Lamarre limite sa discussion de la naissance de la télévision à son statut de symbole du développement de la société de consommation, des communications et de la culture de masse ${ }^{27}$.

En comparaison avec la position des Jones, Bourdon et Lamarre, celle de Léon Dion fait contraste et nous incite à le ranger dans une sorte de sous-ensemble à part. Dans un passage de son étude sur les intellectuels québécois des années cinquante, le politologue ne ménage pas ses mots : «L'événement majeur de la décennie [au Québec], écrit-il, fut peut-être l'implantation de la télévision sous les auspices de la Société Radio-Canada en septembre $1952^{28}$. » Dion défend donc une opinion nettement plus proche du discours dominant que ne le font Jones, Bourdon et Lamarre. D'ailleurs, si plusieurs auteurs présument de la grande influence de l'avènement de la télévision au Québec, comme nous l'avons vu précédemment, Dion est à notre connaissance le seul analyste à proposer une lecture aussi généreuse, à aller aussi loin qu’à en faire le principal événement de la décennie ${ }^{29}$. Mais là où il se démarque de tous les autres, c'est sur le sens à donner à cet événement. Dion - et c'est là l'un des intérêts de sa position - évite de se complaire dans les clichés accrédités et suggère que l'impact de la télévision s'est d'abord fait sentir dans une espèce de renversement des repères spatio-temporels usuels. «La télévision, soutient-il, a le pouvoir de réduire le temps, de le comprimer dans l'instant présent tandis qu'elle familiarise avec un espace qui s'étend jusqu'aux confins du monde. Il s'agit d'une inver- 
sion du rapport immémorial du temps et de l'espace vécus [...] dans l'approche concrète d'une conscience située ici et maintenant $\mathrm{t}^{30}$. $\mathrm{C}^{\prime}$ 'est donc tout le rapport ancien à la temporalité, à la tradition et à la mémoire que la télévision aurait contribué à bouleverser.

En procédant à ce survol, nous ne perdons pas de vue que la fonction de la synthèse est en quelque sorte d'établir l'inventaire des connaissances; elle doit rendre compte de la production existante sur un sujet donné et non pas apporter un savoir neuf. Forcément, si une attention minimale a jusqu'ici été accordée à l'histoire de la télévision au Québec, il ne saurait être question de tenir pour « fautifs » les auteurs de synthèse qui s'en tiennent à peu de choses. Nous désirons quand même insister sur l'espèce de désinvolture, d'indifférence, voire de négligence, avec laquelle ils abordent le rôle de la télévision dans la contestation des années cinquante. Il y a, nous le répétons, une étonnante contradiction entre 1) le poids considérable que la télévision aurait eu, selon plusieurs de ces synthèses, à titre d'agent de contestation du régime et de conscientisation sociale, et 2) la faible importance que cette question occupe dans l'échelle des préoccupations et des questionnements de l'historien québécois. S’il n'est pas vraiment du ressort des synthèses d'ajouter à la connaissance, il est en revanche étonnant, précisément parce qu'il y a dans chaque synthèse un effort pour dresser un état de question, qu'aucune ne souligne les carences historiographiques sur le thème de la télévision - comme le fait Jean de Bonville dans sa contribution au Guide d'histoire $d u$ Québec ${ }^{31}$ — ni n'utilise le travail d'un Paul Rutherford, par exemple. Quand les locuteurs du discours historien dominant que nous avons cités abordent la question de la télévision et de la contestation idéologique dans le Québec des années cinquante, ils ne soulignent jamais qu'au fond, il ne convient pas d'avoir 
les certitudes trop assurées parce que le travail de démonstration reste encore à faire pour une bonne part.

En somme, comme on peut le voir à la lumière de ces quelques exemples, l'historien qui aborde la question de l'importance politique et idéologique de la télévision dans le Québec des années cinquante a tendance à s'en tenir à des «évidences » qui ne sont en réalité que des assertions à la portée non vérifiée. Manifestement, ce que déplorait Gérard Laurence en 1982 vaut encore de nos jours : le souci de pousser les analyses sur cette question fait toujours défaut.

L'attitude contradictoire de la communauté historienne québécoise à l'endroit de la télévision exprime clairement la pertinence d'enquêter sur son origine autant que sur la nature et la portée de sa contribution au déclenchement de la Révolution tranquille. Dans cet esprit, deux hypothèses pourraient guider la recherche future.

La première part du principe que certaines idées, à force d'être constamment répétées, en viennent à s'imposer en tant que vérités " axiomatiques ", n'ayant nul besoin d'être démontrées parce que trop évidentes. La constante réitération de l'influence concrète et considérable exercée par la télévision sur la modernisation des mentalités au Québec a profondément enraciné dans les esprits une conviction à cet effet. La représentation construite de la télévision naissante, de son rôle politique, idéologique ou culturel et de ses effets au sein de la société civile s'est confondue avec le réel non enquêté, au point que celui-ci, de nos jours, ne parait plus pouvoir avoir été autre ${ }^{32}$. La seconde hypothèse, à l'opposé, postule que l'historien a eu raison, toutes ces années, de ne pas trop insister sur la problématique de la télévision naissante au Québec. Bien que celle-ci paraisse négligée à la lumière du bilan que traçait Marcel Dubé (et repris sous une forme ou une autre par de nombreux commentateurs après lui), son importance demeure secondaire. Car 
ce que l'exposé de Dubé traduisait d'abord et avant tout, c'était moins l'importance réelle de la télévision naissante que l'envoûtement exercé par cette nouveauté technologique sur ses premiers adeptes.

Dans le premier cas, en contribuant à départager la représentation et le réel, la recherche approfondie pourrait expliquer, par delà les scénarios poussifs, comment la télévision est parvenue à remplir entre 1952 et 1960 le rôle d'agent de contestation qu'on lui prête. Dans le second, la recherche viendrait peut-être au contraire confirmer la justesse de l'attitude de l'historien québécois. Ce faisant, elle permettrait de corriger le tir dans les synthèses à venir.

\section{Sur une poignée d'outils à la disposition de futurs prospecteurs}

Un demi-siècle après l'avènement de la télévision au Québec, que sait-on vraiment à ce sujet? Quelques jugements hâtifs, quelques opinions tranchées reprises un peu mécaniquement d'une publication à l'autre tiennent souvent lieu d'analyse. Pourtant, sous cette surface opaque perce un certain savoir. Bien qu'aucune étude de fond n'ait encore été réalisée en ce domaine, une somme de travail a déjà été abattue depuis vingt-cinq ans par quelques chercheurs québécois et celle-ci gagnerait à se voir relayer et replacer dans le contexte plus large qu'offre la synthèse : éclairé par des faits de nature autre, ce travail saurait peut-être à son tour éclairer ces mêmes faits d'une lumière nouvelle. Quand l'historien se décidera enfin à creuser la question du sens à donner à l'implantation de la télévision au Québec, il pourra compter sur quelques outils qui lui faciliteront le travail.

Gérard Laurence a tiré deux articles de sa thèse de doctorat sur les premiers programmes à la télévision québécoise. Parus en 1978 et 1982, ces textes adoptent une démarche des- 
criptive, presque ethnographique. Cela est tout particulièrement vrai du premier des deux qui s'attarde à restituer, à travers ses multiples étapes politiques et bureaucratiques, la naissance et les balbutiements initiaux de la télévision de Radio-Canada ${ }^{33}$. Le second, déjà cité, s'intéresse pour sa part aux premières émissions d'affaires publiques de la société d'État (formules, moyens, contenus, invités, etc.) et propose un certain nombre d'éléments fort intéressants sur lesquels nous aurons l'occasion de revenir ${ }^{34}$.

Dans un article du milieu des années quatre-vingt, JeanPierre Desaulniers a présenté quelques pistes de réflexion sur les rapports entre la télévision et les communautés d'appartenance à partir du cas du Québec ${ }^{35}$. Il y a notamment rappelé avec pertinence qu'à l'encontre de ce qui s'est produit dans de nombreux pays, « les intellectuels n'ont pas boudé la télévision au Québec ; ils s'en sont emparés radicalement pendant les premières années ${ }^{36} \%$. Alors qu'un ministre de la $I^{e}$ République française pouvait encore qualifier la télévision de "petit guignol pour distraire mon chauffeur ${ }^{37}$ ", au Québec à la même période, la télévision devenait un enjeu symbolique en rapport avec un programme de résistance au régime en place. Que le degré de succès des résistants soit difficile à déterminer ne change rien à l'affaire : les intellectuels d'ici - le réalise-t-on toujours, et est-on conscient des conséquences de cela sur la mémoire de la télévision construite après coup par ces derniers ? - comptèrent parmi les premiers à imaginer non seulement tout le potentiel de pédagogie politique de ce moyen de communication sans pareil, mais également, en tant que communauté désireuse de faire advenir sa propre véritée ${ }^{38}$, tout son pouvoir de promotion sociale.

Ailleurs, dans une petite synthèse sur la fiction sérielle québécoise, Desaulniers a partiellement abordé la problématique du lien entre télévision et communauté d'appartenance, 
mais cette fois par le biais de l'imaginaire téléromanesque ${ }^{39}$. Selon lui, trois téléromans se distinguent des autres émissions des années cinquante : La famille Plouffe, Le survenant et Les belles histoires des pays d'en haut. Plus accomplies que sous leur forme romanesque parce que dénuées d'interférences cléricoduplessistes (la Société Radio-Canada est un organisme fédéral), ces créations télévisuelles proposèrent aux Canadiens français un renouvellement des références culturelles générales qui leur avaient été imposées jusqu'ici et des représentations canoniques de soi et de l'autre qui étaient toujours en vigueur. L'accent mis sur la mutation de l'esthétique et des sensibilités artistiques rapproche Desaulniers de ce que disait Richard Jones, mais sa perspective se veut plus large et son argumentation bien mieux étoffée.

Des artisans de la télévision naissante ont eux aussi suggéré que celle-ci avait eu des répercussions profondes, mais pas nécessairement détectables à première vue. C'est notamment le cas de René Lévesque et de Gérard Pelletier, tous deux animateurs d'émissions d'affaires publiques.

Au sujet de Point de mire, Lévesque confie dans ses mémoires : "Selon les experts en programmation, rien de plus mort-né que cette idée de faire entrer chez les gens, après Séraphin et Donalda [du téléroman Les belles histoires des pays d'en baut], les vraies histoires des pays lointains ${ }^{40}$. » En 1956, préparant sa toute première émission qui allait porter sur la crise du canal de Suez, Lévesque se demanda comment intéresser le grand public à des choses si étrangères. La solution qu'il imagina fut de s'adapter à la fiction sérielle et de doter ses exposés d'une structure narrative qui les rendrait capables de mieux rejoindre le public :

Si éloignées fussent-elles de Saint-Denis et Sainte-Catherine, les grandes secousses du monde ne seraientelles pas au moins aussi captivantes, peuplées qu'elles 
étaient de personnages ultra-dramatiques et pleines de rebondissements plus étonnants que tous ceux de [Roger] Lemelin ou de [Claude-Henri] Grignon ? Le fil conducteur était là. Non sans expliquer, évidemment, il fallait d'abord raconter et tirer des faits, en tâchant de ne pas les fausser, un récit où il y aurait le suspense ${ }^{41}$.

Si Point de mire favorisa comme on le croit l'ouverture des esprits au monde extérieur, c'est donc peut-être grâce à la fiction sérielle. Déjà reine des ondes à cette époque, elle aurait partiellement soumis le journalisme télévisé d'un René Lévesque à quelques-unes de ses règles propres. Il faut tout de même se garder de gonfler la portée de cette anecdote, aussi sympathique soit-elle. La narration dramatique inspirée de la fiction sérielle n'a jamais submergé le champ de la pratique et du discours journalistiques, ni à la télévision ni ailleurs.

Pour sa part, Gérard Pelletier insiste sur la responsabilité de la jeune télévision dans l'émergence d'un sentiment fortifié de confiance en soi parmi les Canadiens français. Animateur des Idées en marche à compter de 1954, Pelletier jette dans ses mémoires un regard sur la télévision empreint d'une certaine nostalgie. «À l'automne 1952, raconte-t-il trois décennies plus tard, un événement culturel de première importance se produisit au Québec. Pour la première fois dans notre histoire, un mécène de grande taille s'adressait à tous ceux qui savaient écrire ou parler ${ }^{42}$. » Le sentiment d'être capable de faire les choses correctement, soutient Pelletier un peu plus loin, a pu se développer, car il n'existait pas de modèles étrangers auxquels se comparer (et par rapport auxquels se désoler). Il n'y avait pas davantage de regards externes à redouter. Les artisans de la télévision inventèrent leurs propres manières de faire et soumirent leur travail au jugement de compatriotes sans préjugés :

Au royaume neuf de la télévision, il n'existait pas de classiques. [...] À ce moment précis de notre évolution 
culturelle, il valait mieux qu'il en fût ainsi, du moins pour un temps: nous avions besoin par-dessus tout de confiance en nous-mêmes et de quelque répit dans la course au chef-d'œuvre impérissable. Je ne crois pas qu'on en découvre jamais, de ces œuvres éternelles, dans les archives de notre télévision à ses débuts. Mais on y trouvera le témoignage d'un défoulement collectif extraordinaire, d'un moment de création (et de récréation...) comme nous en avons rarement vécu ${ }^{43}$.

Sans toutefois les nier, Léon Dion cherche à nuancer certains des effets présumés de la télévision des années cinquante, et notamment celui, fréquemment évoqué, de l'élargissement des horizons intellectuels. Radio-Canada montra certes aux Canadiens français des situations d'un peu partout à travers le monde. Mais de quoi celles-ci pouvaient-elles bien émaner? "Le prochain épisode se produisant ici ou à l'autre bout du monde les dispens[ait] de se poser ces questions ${ }^{44}$ ", conclut-il un peu sévèrement. Néanmoins, en dépit de cette sévérité, les interrogations de Dion nous apparaissent tout à fait recevables, et nous serions d'ailleurs tenté d'en ajouter une de notre cru : même transmis par un bon vulgarisateur comme René Lévesque, jusqu'à quel point les événements survenant ailleurs dans le monde pouvaient-ils faire sens pour une population sous-scolarisée et donc faiblement équipée sur le plan intellectuel pour saisir les tenants et aboutissants d'enjeux aussi complexes que ceux de la politique internationale ${ }^{45}$ ?

Léon Dion rappelle également la relation très différente que les détenteurs du pouvoir et les intellectuels contestataires vivaient avec la télévision. Les premiers la percevaient comme une menace potentielle et refusaient la plupart du temps d'y apparaître (Maurice Duplessis lui-même la fuyait comme la peste). À l'opposé, elle permettait aux seconds de se faire connaître, de dialoguer entre eux et de diffuser leurs idées jusqu'alors confinées à des revues confidentielles et des colloques 
fermés. La télévision faisait pour eux figure de «soutien incommensurable ${ }^{46}$ ", écrit l'auteur dans un passage qui rejoint ce que mentionnait ailleurs Jean-Pierre Desaulniers. «Sans elle, se demande Dion, qu'auraient été les carrières de Pierre Trudeau, de Gérard Pelletier, de René Lévesque, de Jeanne Sauvé, d'André Laurendeau ${ }^{47}$ ?» Dit autrement, qui des intellectuels ou de la société a le plus profité de la télévision dans les années cinquante?

Prudent dans son évaluation du phénomène télévisuel, Gaston Gauthier en a lui aussi appelé à l'historien pour mieux comprendre les multiples facettes de la télévision et son impact social. Dans un petit article, Gauthier se demande si, au fond, évoquer l'impact profond de la télévision sur la transformation des mentalités, son rôle de "libérateur", son statut de "fenêtre sur le monde », n'équivaut pas à faire voir les enjeux de toute une époque à travers le prisme réducteur d'un combat mythique entre les Lumières et les Ténèbres ${ }^{48}$. Lidée que la télévision ait pu être tout cela accentue naturellement celle, concomitante, de l'existence d'une Grande Noirceur et d'un isolement quasi complet des esprits éclairés au sein de la société québécoise.

La question que soulève Gauthier est celle du rapport entre passé vécu et passé construit. Peut-on, ou doit-on, considérer que l'attribution usuelle d'effets libérateurs à la télévision naissante relève, au moins en partie, du processus de construction d'une nouvelle mémoire collective entrepris après 1960 par ceux-là mêmes qui s'étaient opposés au régime duplessiste ? Suivant cette approche, la mémoire particulière de la télévision aurait été instrumentalisée dans la perspective de l'opposition - systématique et fondamentale à l'identité du Québec contemporain, comme Jocelyn Létourneau l'a montré dans une série d'articles ${ }^{49}$ - entre Grande Noirceur et Révolution tranquille. S'insérant à merveille dans le schéma dichotomique qui 
oppose le temps de Maurice Duplessis à celui de Jean Lesage, la télévision naissante, ses contenus et sa technologie auraient tout naturellement été catapultés par ses premiers utilisateurs et bénéficiaires, les intellectuels, dans le camp du progrès idéo$\operatorname{logique}^{50}$.

Notons, dans la foulée de cette observation, que les narrations du passé québécois mettent peu de temps à rendre la télévision insignifiante. Les schémas interprétant la période post1960 qui ont été proposés jusqu'ici ne lui accordent aucune importance politique, culturelle, idéologique ou autre. Le contraste avec les schémas valant pour les années cinquante est donc absolument frappant. C'est un peu comme si, à la charnière de 1960, après la mort du tyran et l'avènement de l'Âge d'Or, l'historien contemporain, n'en ayant plus besoin, s'était mis à regarder la télévision de si haut qu'il avait fini par la perdre de vue. Il n'entendait plus guère, dès lors, que son incessant et navrant bavardage public, et encore. Déjà, au milieu des années cinquante, Gérard Pelletier attribuait à la télévision le pouvoir d'éduquer les citoyens aussi bien que celui de les engourdir intellectuellement. À en juger par ce qui se dit et s'écrit souvent au sein du discours social, plusieurs, et pas seulement parmi les historiens, semblent croire que la télévision a massivement contribué à l'éveil de la population dans les années cinquante, avant de soudainement, mais irrémédiablement, se mettre à l'abrutir. Là loge peut-être une raison de cette perte d'intérêt pour elle. L'idée voulant que la production télévisuelle des années cinquante ait été nettement meilleure que celle d'aujourd'hui reste d'ailleurs bien vivante de nos jours ${ }^{51}$.

Parmi les nombreuses questions que soulève la contradiction entre l'influence politique non négligeable que l'historien prête à la télévision des années cinquante et la faible attention critique qu'il lui accorde, quelques-unes sont sans doute destinées à demeurer vaines, faute d'une documentation ap- 
propriée à soumettre à l'analyse. Mais en croisant les perspectives intellectuelles, en combinant les méthodes d'enquête, il est possible que les réponses à certaines de ces questions permettent de mieux évaluer le phénomène. Ce que l'on croit savoir de la naissance de la télévision et de ses premiers effets sur le renouveau des mentalités canadiennes-françaises pourrait alors se voir confirmer ou infirmer, ou simplement nuancer.

Par exemple, l'idée que les premiers publics furent captifs de leurs postes de télévision s'est rapidement imposée à plus d'un observateur. Dès même les années cinquante, alors que la société apprivoisait encore l'invention, cette idée vaguement catastrophiste s'est mise à circuler. Gérard Pelletier décrivait ainsi le téléspectateur comme

[...] prisonnier de son foyer, de son fauteuil, de l'image qu'on lui offre. Et comme tout captif, il est désarmé, passif, livré sans défense à ceux qui ont capté son attention. Mais comme un prisonnier aussi, il est recueilli, disponible, tout entier tourné vers ces images qu'on lui présente, seule fenêtre de son cachot $^{52}$.

Simpliste, cette idée, comme d'ailleurs n'importe quelle idée simpliste, s'est avérée très résistante puisqu'on la retrouve encore dans la littérature contemporain ${ }^{53}$. Mais jusqu'à quel point résisterait-elle à l'analyse plus poussée ?

Paul Rutherford explique à ce propos que l'écoute de la télévision, dans les premières années, ne se faisait pas nécessairement de manière passive et recueillie, pour reprendre les termes de Pelletier. Des recherches menées à cette époque aux États-Unis ont montré que le téléspectateur n'était pas toujours préoccupé en priorité par ce que son téléviseur lui présentait. Loin d'être un prisonnier, il partageait en fait son attention avec des activités alternatives. Les discussions et le vaet-vient abondaient pendant les programmes. Regarder la télévision représentait souvent une activité familiale, une pratique 
sociale rassembleuse, une occasion de resserrer les liens avec les autres ${ }^{54}$. Par ailleurs, le rapport à la télévision se vivait dans un état d'esprit particulier. Objet de curiosité, la «boîte à images » fascinait en soi autant sinon plus que ses contenus variés, à tout le moins dans les premiers temps. La naissance de la télévision, il ne faut pas l'oublier, s'inscrit dans une période de grand progrès technique et technologique, mais aussi de grande fascination pour la technique et la technologie en général. Enfin, la télévision offrait déjà un potentiel de divertissement facile. La "présence » que chaque téléviseur assurait au sein du foyer, les images animées qu'il diffusait, la musique qu'il faisait entendre concourraient toutes à favoriser une certaine écoute insouciante (casual). Rutherford en conclut que « regarder la télévision constituait l'un des moyens les plus accessibles d'échapper à l'ennui du vécu quotidien ${ }^{55}$ ». Pour toutes ces raisons, il paraît difficile d'accréditer sans nuance la thèse qui fait de la télévision une sorte de prison dorée pour citoyens dépourvus. Dans la même veine, il faut se demander ce que la popularité de Point de mire et des Idées en marche - attestée par la cote d'écoute $^{56}$ — nous révèle vraiment de l'intérêt des Canadiens français pour les affaires publiques et de leur compréhension des dossiers présentés et débattus devant eux $^{57}$.

Les résultats de l'élection québécoise de 1960 devraientils eux aussi inciter l'historien à réévaluer l'influence sociale présumée de la télévision ? L'un des topiques du récit presque hégémonique de la Révolution tranquille veut que le scrutin du 22 juin ait été le moment où l'ensemble du Québec, épuisé par quinze années de duplessisme rétrograde, a choisi de «briser ses chaînes » et de combattre les sources de son aliénation passée en adhérant au vaste programme de réformes proposé par les libéraux. Et pourtant, avec $51 \%$ des voix contre $47 \%$ accordées à l'Union nationale, le Parti libéral est loin d'avoir créé un raz-de-marée. Sa victoire ne peut même pas être tenue 
pour l'expression d'une volonté consensuelle à l'échelle de la société. L'entrecroisement d'une foule de facteurs explique le score relativement élevé de l'Union nationale, parmi lesquels, nous n'en doutons pas, figurent en bonne place le patronage, la corruption et une panoplie d'autres pratiques électoralistes douteuses. Cela dit, force est d'admettre qu'après une décennie ou presque d'appels au changement (sous forme de critique de l'Union nationale, de remises en question radicales de l'ordre établi et de contestation politique) lancés sur les ondes de la Société Radio-Canada - infestée de gauchistes, pestait Robert Rumilly en son temps ${ }^{58}$ ! — ces résultats soulèvent certaines questions en rapport avec ce que le public pouvait retenir des palabres des intellectuels contestataires une fois les émissions terminées.

Dans le contexte de cette élection, Susan Mann Trofimenkoff avance une hypothèse en rapport avec l'influence exercée par la télévision. Selon elle, le remplacement de Georges-Émile Lapalme par Jean Lesage à la tête du Parti libéral s'expliquerait au moins partiellement par le fait que celui-ci passait mieux au petit écran que celui-lă ${ }^{59}$. On connaît des exemples où la télévision s'est révélée un acteur de premier plan dans une campagne électorale (le fameux débat entre Richard Nixon et John F. Kennedy en 1960 est le cas le plus souvent cité). Il paraît très plausible que les libéraux aient bel et bien eu cette préoccupation en tête en préparant la campagne de 1960. Après tout, la télévision ayant été depuis sa naissance, sur les plans politique et idéologique, l'affaire des opposants au régime, il serait logique de croire que le PLQ en a tenu compte dans la mise au point de sa stratégie de campagne. Mais dans la mesure où l'Union nationale boudait la télévision, il sera toujours ardu d'évaluer l'avantage concret que la télégénie supérieure de Lesage a pu lui apporter. Pour tout dire, il se pourrait que les clefs de la victoire libérale aient été la mort opportune 
de Duplessis en septembre 1959 et la personnalité fade et sans charisme de celui qui était alors chef de l'Union nationale, Antonio Barrette ${ }^{60}$.

\section{$* * *$}

Cette merveille technologique, nota un jour Gérard Pelletier, nous nous y sommes babitués avant de l'avoir comprise. [...] La familiarité camoufle si bien l'ignorance qu'elle endort pour toujours le besoin d'apprendre, qu'elle engourdit le remords d'ignorer. En sommesnous arrivés là dans nos rapports avec la télévision? Cela reste à voir ${ }^{61}$.

C'était en 1956, soit bien avant que Pelletier, rédigeant ses souvenirs, ne célèbre avec exubérance la « révolution idéo$\operatorname{logique}^{62}$ » que la télévision fit supposément advenir dans le Québec des années cinquante. Mais le propos n'a rien perdu de sa pertinence aujourd'hui.

En vérité, la part exacte de "révolution idéologique » dans le legs de la télévision naissante ne se laisse pas déterminer aisément. Alors qu'environ $90 \%$ des foyers québécois étaient équipés d'un téléviseur en 1960, certaines conditions pour qu'une telle révolution ait lieu semblent avoir été réunies. À la fois agent et témoignage de modernité, la télévision servit jusqu'à un certain point de métaphore au complexe processus d'intégration continentale par lequel la société québécoise des années cinquante s'arrima à une vitesse accrue au reste de l'Amérique du Nord ${ }^{63}$. Mais au delà de ce premier jugement, plusieurs questions attendent encore de trouver réponse, des questions qui exigent que l'historien prenne en compte à la fois le passé vécu et le passé raconté, la réalité de l'ayant-été et sa mémoire construite a posteriori.

"Quel aura été l'effet de ces vues animées sur des générations auxquelles on les sert depuis une trentaine d'années?, 
s'est demandé René Lévesque au milieu des années quatre-vingt. Bien malin qui pourrait l'évaluer avec certitude, ou mieux, bien présomptueux qui prétendrait le faire ${ }^{64}$. » $\mathrm{Ce}$ point de vue pessimiste et résigné adopté par Lévesque nous apparaitt irrecevable parce qu'il condamnerait d'avance, s'il fallait y souscrire, la démarche de l'historien à la stérilité. Il est au contraire à souhaiter que dans un avenir rapproché, celui-ci canalise une part de plus en plus grande de son temps et de ses ressources en direction de cette problématique.

Pour que cela se produise, trois développements s'avéreront nécessaires. Au premier chef, il faudra que s'atténue la sujétion de l'historien à ce que l'on nommera, faute d'un mot plus juste, la «tyrannie » de la source écrite. Jusqu’ici, les références culturelles premières de l'historien québécois ont toujours été littéraires, et jamais audiovisuelles, de sorte que les choix de sources qu'il a opérés en ont évidemment porté la marque. Les sources audiovisuelles - la télévision, le cinéma, la radio et, à sa façon, l'univers virtuel de la Toile - se caractérisent par le mouvement, la fluidité, le changement. En cela, il est aisé de comprendre qu'au sein de la communauté historienne québécoise, ces sources apparaissent bien éloignées du terrain familier et sécurisant des données fixées sur le papier une fois pour toutes. Nous n'ignorons pas que l'historien met de plus en plus l'iconographie et les sources orales à contribution dans ses travaux, mais il semble que ce soit souvent lorsque la documentation écrite ne lui suffit pas qu'il les appelle en renfort. Mille images (ou mille paroles) valent généralement moins qu'un mot écrit. La "tyrannie» de l'écrit à laquelle les positivistes s'étaient autrefois assujettis, certains d'être guidés vers la Vérité historique en échange de leur soumission, n'a donc pas complètement disparu, bien que les illusions d'accéder à la substance de l'ayant-été qui l'accompagnaient, elles, ont évidemment été abandonnées depuis longtemps. 
Un peu dans la même veine, il faut prendre en considération que la communauté historienne québécoise actuelle est friande d'histoire sociale et qu'elle dirige une bonne part de son attention vers des problèmes pouvant se résoudre à l'aide de données quantifiables. Or, la nature même des sources qu'il faudrait prioritairement utiliser pour produire une histoire de la télévision rend difficilement opérantes les méthodes propres à l'histoire sociale.

Il y a malgré tout de l'espoir. Ayant grandi avec la télévision, puis avec Internet, les prochaines générations d'historiens conviendront peut-être qu'une émission de télévision suivie par des millions de Québécois pendant des mois, voire des années, mérite quelque reconnaissance dans le contexte d'une réflexion sur les mentalités, la culture, l'imaginaire ou encore l'identité $e^{65}$.

En second lieu, il faudra également que la culture de masse, particulièrement lorsqu'il s'agit de divertissement, cesse de faire l'objet du mépris, plus souvent larvé qu'explicitement affirmé, de ceux qui se donnent le mandat de réfléchir sur la société et d'en rendre le passé intelligible. Ce changement n'est toutefois pas pour demain, car l'habitude des intellectuels d'ici de jeter un regard condescendant sur le divertissement populaire est ancienne. En rien propre à la société québécoise ou canadienne-française, cette habitude, chez nous, renvoie en le transcendant au vieux combat pour la survivance.

Tout en reconnaissant simplifier quelque peu la matière complexe du passé aux fins de son propos, Gérard Bouchard a dégagé, à un premier niveau d'analyse, deux grands types de cultures en construction dans le Canada français du XIX siècle $^{66}$. La première, celle des élites socioculturelles, était une culture dont les références venaient à peu près exclusivement d'Europe, et surtout de France. La seconde, celle des classes populaires, se voulait plutôt ouverte sur la réalité continentale 
et intégrait volontiers des apports variés provenant surtout des États-Unis. Bouchard parle d'ailleurs de cette opposition en termes de culture de la continuité et de culture des recommencements ${ }^{67}$.

Les élites n'ont pas rejeté en bloc la culture populaire, tant s'en faut. Au contraire, elles ont voulu voir dans cette culture une espèce de « dépôt sacré des vieilles coutumes et traditions françaises ${ }^{68}$ ", un fondement de la nation, le lieu d'une authenticité nationale, se conformant en cela à l'attitude dominante en Europe au même moment ${ }^{69}$. Toutefois, elles ont eu tendance à ignorer, ou alors condamner, tout ce qui, dans cette culture, ne s'accordait pas avec l'idéal de continuité de la vieille France en Amérique. S'adjugeant un rôle « de pionniers et de pédagogues de la culture », écrit encore Bouchard, ces élites entreprirent de «combler le vacuum culturel - entendons, européaniser une culture populaire nord-américaine, aménager et consolider l'espace distinct ${ }^{70} »$. Posant la nation canadienne-française et sa culture comme différentes de ses voisines par leur essence, et postulant que cette essence tenait tout entier dans son legs français, les élites ont conséquemment vu d'un fort mauvais œil les pratiques culturelles populaires importées des États-Unis. Comme autant de chevaux de Troie, celles-ci semblaient inoculer de dangereux poisons à la nationalité et en compromettre l'unité, voire la pureté ${ }^{71}$. Le divertissement populaire fut sévèrement sanctionné. Le folklore religieux ou inspiré du terroir, par exemple, reçut l'imprimatur des clérico-nationalistes, mais le cinématographe, bien qu'il fit courir les foules de Montréal à son avènement en 1896, n'eut pas la même chance. Dans le même esprit, la bonne chanson fut rapidement distinguée de la mauvaise, les loisirs acceptables de ceux qui ne l'étaient pas. Bref, il y eut la bonne culture et la culture malsaine $^{72}$. Toutes les pratiques culturelles de divertissement dénoncées par les élites socioculturelles avaient en com- 
mun leur accessibilité au plus grand nombre, leur nouveauté et, la plupart du temps, leur origine étatsunienne.

Aujourd'hui, les condamnations pontifiantes du divertissement populaire n'ont plus autant la cote. Le divertissement à la manière étatsunienne imprègne tellement la société québécoise et influence à tel point ses propres créations que les attitudes à son endroit, en règle générale, se sont adoucies. Ce qui est resté aussi vivace parmi les élites socioculturelles, par contre, c'est la croyance en leur fonction de "pédagogues de la culture », pour reprendre les mots de Bouchard. C'est l'autorité auto-octroyée de déterminer ce qui est méritant et ce qui ne l'est pas. Au sujet de la lecture de loisir et du marché québécois des livres à gros tirage, lesquels reçoivent peu d'attention de la part des intellectuels, Denis Saint-Jacques a ainsi écrit que " la culture élitaire [dans le domaine littéraire] est alors promue nationale et la culture de masse rejetée comme étrangère ; c'est un tour de passe-passe qui ne trompe peut-être que les intellectuels qui veulent y croire ${ }^{73} »$. Sur la base d'un tel mépris pour la culture de divertissement populaire, ou d'une telle ignorance volontaire de cette culture, certains, dans le but affirmé d'analyser les sensibilités actuelles de la collectivité québécoise, vont alors se tourner exclusivement vers les romans « songés » tirés à deux cent cinquante exemplaires et lever le nez sur des œuvres vendues à cent mille copies. Autre exemple, mais cette fois dans le domaine de la musique : il y a quelques années, recensant un essai sur la construction du personnage de Céline Dion en figure rassembleuse pour les Canadiens français du Qué$\operatorname{bec}^{74}$, le critique d'un quotidien montréalais, loin d'aborder le livre avec l'ouverture d'esprit qu'on serait en droit d'attendre d'un recenseur et d'un intellectuel, a plutôt écrit que l'objet du livre avait de quoi «agresser ${ }^{75} »$. C'est comme si, avec cette enquête osant associer au problème névralgique de l'identité québécoise un tel personnage - honni par la majorité des in- 
tellectuels canadiens-français du Québec parce que trop étroitement collé, selon eux, sur la culture de masse étatsunienne, avaient été franchies les limites non seulement du dicible, mais aussi du pensable. De telles postures par rapport à la culture de divertissement de masse donnent une idée des obstacles qui se dressent devant la télévision sur le chemin de la respectabilité culturelle et universitaire. En effet, pourrions-nous demander, si regarder la fiction sérielle est une perte de temps, comme tant d'intellectuels semblent le penser, pourquoi l'étudier ne le serait-il pas également ? Si la télévision populaire est abrutissante, en quoi sa connaissance "scientifique " viendrait-elle enrichir la collectivité ?

Enfin, il existe une cause additionnelle et plus profonde à la réticence de l'historien à revenir sur le rôle et les répercussions politiques de la télévision dans le Québec des années cinquante. Jocelyn Létourneau a brillamment soutenu que le récit de la Grande Noirceur et de la Révolution tranquille qui s'est élaboré dans les années soixante et soixante-dix était un récit identitaire parce qu'il visait avant toute chose à raconter le parcours de ses promoteurs, la technocratie ${ }^{76}$. Nous avons pour notre part suggéré que le microrécit de la télévision, même dans son état superficiel actuel, obéissait à la même logique et servait d'abord à perpétuer la mémoire de la technocratie ${ }^{77}$. La lecture des synthèses d'histoire du Québec ne laisse planer aucun doute à ce sujet: la télévision ne fut historiquement importante que le temps que la technocratie émergente en eut besoin pour se faire connaitre et diffuser sa critique sociale. Car dès lors qu'elle s'empara des rênes du pouvoir, dès lors qu'elle n'eut plus besoin de la télévision, elle en oublia presque son existence $^{78}$. La télévision perdit-elle sa capacité d'ouvrir les horizons intellectuels du public après 1960 ? Cessa-t-elle de faire entrer le monde dans les salons? S'arrêta-t-elle de transmettre le goût des idées et des débats? À s'en remettre au discours 
dominant dans les synthèses, il faudrait croire que oui. La proposition est absurde, mais la récuser en réévaluant le récit accrédité de l'avènement de la télévision compromettrait celui, plus global, que la technocratie a élaboré au sujet de son propre avènement en tant que communauté de sens. Tant que son récit identitaire réussira à se faire accepter comme unique récit collectif du Québec, la même interprétation risque très fort de prévaloir.

Ce n'est pas seulement l'histoire de la télévision au Québec qui reste à faire. C'est aussi la lutte aux préjugés à son endroit.

\section{NOTES}

${ }^{1}$ L'auteur tient à remercier les évaluateurs de Mens pour leurs commentaires sur une première version de ce texte.

2 Marcel Dubé, «Dix ans de télévision », texte d'une conférence donnée à l'occasion du troisième Congrès du Spectacle, Montréal, 4 mai 1962, reproduit dans Cité libre, XIII année, nº 48 (1962), pp. 22-26.

${ }^{3}$ Ibid., p. 24.

+ Gérard Laurence, «Le début des affaires publiques à la télévision québécoise, 1952-1957 », Revue d'bistoire de l'Amérique française, vol. 36, n 2 (1982), p. 213.

${ }^{5}$ Paul Rutherford. When Television Was Young. Primetime Canada, 1952-1967. Toronto, University of Toronto Press, 1990.657 p. Quoique fort utile, cette étude se consacre néanmoins au premier chef à la télévision et aux publics de langue anglaise. Une dizaine d'années plus tôt, Frank W. Peers a lui aussi publié une histoire de la télévision au Canada. Comme Rutherford, il s'intéresse surtout au Canada hors Québec et au réseau anglais, mais contrairement à lui, il insiste principalement sur les politiques du gouvernement fédéral en matière de télédiffusion. Frank W. Peers. The Public Eye. Television and the Politics of Canadian Broadcasting, 1952-1968. Toronto, University of Toronto Press, 1979. 459 p. Lus en parallèle, ces deux livres offrent une bonne introduction à l'histoire de la naissance de la télévision publique 
canadienne, mais dans laquelle l'importance accordée au volet francophone laisse grandement à désirer.

'Dans ce texte, le terme historien désigne quiconque aborde le passé sous un angle ou un autre, indépendamment de sa formation académique.

${ }^{7}$ Susan Mann Trofimenkoff. Visions nationales. Une histoire du Québec. Montréal, Trécarré, 1986. 455 p. (Traduction de The Dream Of Nation. A Social and Intellectual History Of Quebec. Toronto, MacMillan, 1982. 344 p.) Voir le chapitre XVIII intitulé «Ici Radio-Canada ", pp. 379-399. Il faut préciser que ce chapitre, dans son ensemble, traite du climat de contestation durant les années d'après-guerre et non de la seule télévision. Cela dit, il est intéressant de noter qu'en raison de l'état de la recherche à l'époque, aucun des 22 titres suggérés dans la bibliographie sélective placée à la fin ne porte sur la télévision. Une nouvelle édition, vingt ans après la parution de l'édition originale, ne changerait pas grand-chose à cette situation.

${ }^{8}$ Gilles Gougeon. Histoire du nationalisme québécois. Entrevues avec sept spécialistes, Montréal, VLB éditeur / SRC, 1993. Pp. 111-143. Gougeon ne relève pas l'omission de Desrosiers.

${ }^{9}$ Fernand Dumont et al., dir. Idéologies au Canada français, t. III : 1940-1976. Québec, Presses de l'Université Laval, 1981. 3 vol.

1" Alain-G. Gagnon et Michel Sarra-Bournet, dir. Duplessis. Entre la Grande Noirceur et la société libérale. Montréal, Québec Amérique, 1997. 396 p.

"Ibid., quatrième de couverture.

${ }^{12}$ Jacques Lacoursière. Histoire populaire du Québec, tome IV : De 1896 à 1960. Sillery, Septentrion, 1997. 411 p. ; Jacques Lacoursière, Jean Provencher et Denis Vaugeois. Canada-Québec, 1534-2000. Sillery, Septentrion, 2000. 591 p.

${ }_{13}^{13}$ Paul-André Linteau, René Durocher, Jean-Claude Robert et François Ricard, Histoire du Québec contemporain, t. 2: Le Québec depuis 1930, Montréal, Boréal, 1986, pp. 367-368. La seconde édition, parue en 1989, reproduit le même propos.

${ }^{14}$ Ibid., p. 368.

${ }^{15}$ Ibid., pp. 368-369.

${ }^{16}$ Gilles Laporte et Luc Lefebvre, Fondements bistoriques du Québec contemporain, Montréal, La Chenelière, 1995, pp. 244-245.

${ }^{17}$ Yves Tessier, Histoire du Québec d'bier à l'an 2000. Les fondements historiques du Québec contemporain, Montréal, Guérin, 1994, pp. 204-205. 
${ }^{18}$ Louis Balthazar, Bilan du nationalisme au Québec, Montréal, L'Hexagone, 1986, p. 113. Dans la même veine mais en plus de mots, voir aussi ce que dit Balthazar dans Gougeon, op. cit., pp. 164-165.

${ }^{19}$ Gérard Bouchard, Genèse des nations et cultures du Nouveau Monde. Essai d'bistoire comparée, Montréal, Boréal, 2000, p. 160.

20) Jacques Lacoursière, Une histoire du Québec, Sillery, Septentrion, 2002, p. 162.

${ }^{21}$ Denis Monière, Le développement des idéologies au Québec des origines à nos jours, Montréal, Québec Amérique, 1977, p. 308.

22 Yves Bélanger, Robert Comeau et Céline Métivier, dir. La Révolution tranquille 40 ans plus tard : un bilan. Montréal, VLB éditeur, 2000. $316 \mathrm{p}$.

${ }^{23}$ Stéphane Dion, «Le rôle moteur du gouvernement du Canada dans la Révolution tranquille », dans ibid., p. 56 ; Marc Laurendeau, "L'ébullition culturelle pendant la Révolution tranquille », dans ibid., p. 257.

${ }^{24}$ Jean Hamelin et Jean Provencher, Brève histoire du Québec, $5^{\mathrm{e}}$ éd., Montréal, Boréal, 1987, p. 107. Le service de télévision de la Société Radio-Canada est créé - sur papier — en 1951, mais c'est à partir de 1952 que les émissions commencent.

${ }^{25}$ John A. Dickinson et Brian Young, Brève bistoire socio-économique du Québec, Sillery, Septentrion, 1992, p. 313. (Traduction de A Short History Of Quebec. A Socio-Economic Perspective. Toronto, Copp Clark Pittman, 1988. 306 p.) Le texte de la seconde édition de langue française, parue en 1995, est identique.

${ }^{26}$ Richard Jones. « La remise en question, 1939-1960», dans Jean Hamelin, dir. Histoire du Québec, Montréal, France Amérique, 1976. Pp. 481-483.

${ }^{27}$ Yves Bourdon et Jean Lamarre, Histoire du Québec. Une société nord-américaine, Montréal, Beauchemin, 1998, pp. 203 et 206.

${ }^{28}$ Léon Dion, Québec 1945-2000, t. II : Les intellectuels et le temps de Duplessis, Sainte-Foy, Presses de l'Université Laval, 1993, p. 317. Nous soulignons.

${ }^{29}$ À l'exception, peut-être, de Susan Mann Trofimenkoff. Celle-ci n'est pas aussi explicite et catégorique que Dion, mais la lecture de son livre laisse l'impression qu'elle n'est sans doute pas loin de penser comme lui.

${ }^{30}$ Dion, op. cit., p. 318. 
${ }^{31}$ Jean de Bonville, «Médias de communication », dans Jacques Rouillard, dir., Guide d'histoire du Québec du régime français à nos jours. Bibliographie commentée, Montréal, Méridien, 1991, p. 345.

${ }^{32}$ Frédéric Demers, «Mémoire savante et passé construit : retour sur l'avènement de la télévision au Québec », texte inédit d'une communication présentée au $55^{\mathrm{e}}$ congrès de l'Institut d'histoire de l'Amérique française, Sherbrooke, octobre 2002.

${ }^{33}$ Gérard Laurence. «La naissance de la télévision au Québec, 1949$1953 »$, .Communication information, vol. 2, n 3 (1978), pp. 25-64.

${ }^{34}$ Laurence, "Le début... », loc. cit.

${ }^{35}$ Jean-Pierre Desaulniers. «Télévision et nationalisme ». Communication information, vol. 7, n 3 (1985), pp. 25-36.

${ }^{36}$ Ibid., p. 30.

${ }^{37}$ Cité par Laurence, «La naissance... ", loc. cit., p. 62.

${ }^{38}$ Jocelyn Létourneau. «Le "Québec moderne" : un chapitre du grand récit collectif des Québécois ». Revue française de science politique, vol. 42, $\mathrm{n}^{\circ} 5$ (1992), pp. 765-785.

${ }^{39}$ Jean-Pierre Desaulniers. De La famille Plouffe à La petite vie. Les Québécois et leurs téléromans. Montréal, Fides / Musée de la civilisation, 1996.119 p.

${ }^{40}$ René Lévesque, Attendez que je me rappelle..., Montréal, Québec Amérique, 1986, p. 189.

${ }^{41}$ Ibid., p. 190.

${ }^{42}$ Gérard Pelletier, Les années d'impatience, 1950-1960, Montréal, Stanké, 1983, p. 227.

${ }^{+3}$ Ibid., p. 235.

${ }^{44}$ Dion, op. cit., p. 319.

${ }^{45}$ Sur la question des horizons élargis grâce à la télévision, Gérard Laurence y va lui aussi de quelques mises en garde. Si les émissions d'affaires publiques des années cinquante ont incontestablement contribué à briser un certain silence, elles se sont néanmoins faites dans un climat qu'il qualifie de «liberté surveillée ». L'autocensure des animateurs n'était pas rare et plusieurs sujets restaient tabous. Laurence, « Le début... », loc. cit.

${ }^{46}$ Dion, op. cit., p. 319. 
${ }^{48}$ Gaston Gauthier, "Télévision, mythe et histoire », Traces, vol. 31, $\mathrm{n}^{\circ} 2$ (1993), p. 32.

49 Jocelyn Létourneau. "Histoire du Québec d'après-guerre et mémoire collective de la technocratie ». Cahiers internationaux de sociologie, $n^{\circ} 90$ (1991), pp. 67-87 ; « La mise en intrigue. Configuration historico-linguistique d'une grève célébrée : Asbestos, P.Q., 1949 »,.Recherches sémiotiques, vol. 4, n 3 (1992), pp. 53-71 ; «Le "Québec moderne"... », loc. cit.

${ }^{50}$ Demers, « Mémoire savante... », loc. cit.

${ }^{51}$ Que les émissions de télévision aient été de plus grande qualité n'aurait rien de surprenant, après tout. Chacun sait bien qu'autrefois, les jeunes étaient plus polis, les relations interpersonnelles plus authentiques, les familles plus solidaires, les valeurs meilleures, les joueurs de hockey plus dédiés...

${ }^{52}$ Gérard Pelletier, «Un défi : la télévision », Cité libre, n 15 (1956), p. 9.

${ }^{53}$ Michel Lemieux. L’affreuse télévision. Montréal, Guérin, 1990. 194 p.

${ }^{54}$ Rutherford, op. cit., pp. 449-451.

55 « [...] watching television was one of the easiest ways to escape from real life, especially from the ills of boredom ». Ibid., p. 451.

${ }^{56}$ Laurence, « Le début... », loc. cit.

${ }^{57}$ En considérant la cote d'écoute élevée de ces émissions, il faut garder à l'esprit que dans ces années, il n'y avait qu'une seule chaîne en français. Regarder la télévision, ça voulait dire regarder Radio-Canada. Le téléspectateur n'était pas nécessairement à la merci de son poste de télévision, comme le pensait Gérard Pelletier, mais à moins de comprendre l'anglais et d'habiter suffisamment près de la frontière pour capter les ondes venant des ÉtatsUnis, il était sans nul doute soumis à la Société d'État!

${ }^{58}$ Robert Rumilly, L'infiltration gauchiste au Canada français, Montréal, s.n., 1956, pp. 88-95. Sans aller jusqu'à formuler des pronostics sur ce qu'elle pourrait éventuellement avoir comme conséquences politiques dans un avenir plus ou moins rapproché, Rumilly s'inquiétait tout de même de l'orientation idéologique que la télévision de Radio-Canada, depuis son lancement, avait fait sienne dans ses différentes émissions d'affaires publiques. Déplorant la situation, il écrivit que "les gauchistes aquièrent ainsi une habitude, une vraie technique de la télévision, dont la chance est refusée aux autres ». Son 
inquiétude au sujet des capacités de la télévision de modeler «l'esprit des Canadiens français » était bien réelle. "Il est déjà inconstitutionnel, fit-il valoir, que l'État fédéral accapare une tranche de l'éducation - domaine réservé aux provinces - comme il le fait par le truchement de RadioCanada. [...] La Société Radio-Canada, telle qu'elle fonctionne, est illégale. » Ce n'est pas tout : Rumilly estimait également « intolérable » que la Société d'État, « vivant des deniers du peuple canadien-français, cherche à l'entrâner vers une idéologie contraire à ses traditions et à ses aspirations nationales » (pp. 94-95 passim). Dans ce même esprit, mais avec une perspective davantage religieuse (ou morale) que politique, voir Influence de la presse, $d u$ cinéma, de la radio et de la télévision, $\mathrm{XXXIV}^{\mathrm{e}}$ session des Semaines sociales du Canada (Montréal, 1957), Montréal, Institut social populaire, 1957, et notamment la «Déclaration d'ouverture » du R. P. Joseph-P. Archambault, s.j. (pp. 9-18).

${ }^{59}$ Trofimenkoff, op. cit., p. 398.

(1) Roublard, charismatique, sans scrupules, chapeautant une organisation bien huilée et pouvant s'appuyer sur un réseau de débiteurs politiques qui devraient bien finir, un jour ou l'autre, par lui rembourser leur dette, Duplessis aurait aussi pu jouer la carte du politicien enraciné dans la province pour discréditer Lesage, l'homme d'Ottawa, l'ancien ministre centralisateur. Dans la culture politique québécoise, l'accusation de n'être qu'un pion du gouvernement fédéral peut s'avérer très préjudiciable. Une quarantaine d'années plus tard, Jean Charest l'a d'ailleurs appris à ses dépens. Qu'aurait donc fait Lesage, lui-même plus habile et charismatique que Georges-Émile Lapalme, face à un adversaire comme Duplessis ? Quel dommage qu'il ne suffise pas, dans ce cas-ci, de simplement poser la question pour en avoir la réponse!

${ }^{61}$ Pelletier, loc. cit., p. 4.

${ }^{62}$ Pelletier, op. cit., p. 231.

${ }^{63}$ Marie-Charlotte De Koninck, dir. Jamais plus comme avant! Le Québec de 1945 à 1960. Montréal, Fides / Musée de la civilisation, 1995. 183 p. Voir le chapitre 6 en particulier.

${ }^{64}$ Lévesque, op. cit., p. 161.

${ }^{65}$ L'émergence, il y a dix ou quinze ans, d'une nouvelle sensibilité historique critique de la sensibilité précédente (à qui on doit le récit classique de la Révolution tranquille) donne d'autant plus de raisons d'espérer. Sur cette 
nouvelle sensibilité, voir Stéphane Kelly, dir. Les idées mènent le Québec. Essais sur une sensibilité historique. Québec, Presses de l'Université Laval, 2003. 219 p. ${ }^{66}$ Gérard Bouchard. "Une nation, deux cultures : continuités et ruptures dans la pensée québécoise traditionnelle (1840-1960) », dans Gérard Bouchard, dir., avec la coll. de Serge Courville. La construction d'une culture. Le Québec et l'Amérique française, Sainte-Foy, Presses de l'Université Laval, 1993. Pp. 3-47.

${ }^{67}$ Ibid., p. 6.

${ }^{68}$ Ibid., p. 7.

${ }^{69}$ Ernest Gellner. Nations et nationalisme. Paris, Payot, 1989 [1983]. 208 p.

${ }^{70}$ Bouchard, loc. cit. , pp. 13-14.

${ }^{71}$ Ibid., pp. 18-19.

${ }^{72}$ Sur la manifestation du même phénomène aux États-Unis, à la même époque, voir Lawrence W. Levine. Highbrow / Lowbrow. The Emergence Of Cultural Hierarchy In America. Cambridge, Harvard University Press, 1988. $306 \mathrm{p}$.

${ }^{73}$ Denis Saint-Jacques, «Évolution du marché du best-seller au Québec dans les années 1980 », dans Pierre Lanthier et Guildo Rousseau, dir. La culture inventée. Les stratégies culturelles aux XIX et $X X^{e}$ siècles, Québec, Institut québécois de recherche sur la culture, 1992, p. 273, cité dans Yvan Lamonde, Allégeances et dépendances. L'bistoire d'une ambivalence identitaire, Québec, Nota Bene, 2001, pp. 101-102.

${ }^{74}$ Frédéric Demers. Céline Dion et lidentité québécoise. Montréal, VLB éditeur, 1999. $191 \mathrm{p}$.

${ }^{75}$ Louis Cornellier, « Maitriser Céline », Le Devoir, 17-18 avril 1999, p. D7.

${ }^{76}$ Sur ce concept, voir les articles de Létourneau précédemment cités.

${ }^{77}$ Demers, "Mémoire savante... », loc. cit.

${ }^{78}$ Pourquoi, en effet, les émissions d'affaires publiques depuis le début des années soixante ne reçoivent-elles pas la même considération, ni la même reconnaissance, que Point de mire? Parce qu'elles ne s'intègrent pas au récit identitaire de la technocratie ? Parce que cette dernière n'en fit pas usage dans sa marche vers le pouvoir? Il est vrai que la mémoire de Point de mire bénéficie du mythe de René Lévesque, mais ceci explique-t-il tout? 